\title{
Estratégias de Assistência no Cuidado à Pessoa com Síndrome da Imunodeficiência Adquirida
}

\section{Strategies of assistance in the care of people with Immunodeficiency Syndrome Acquired}

\author{
Karine Lemos Maciel ${ }^{1}$ \\ Viviane Marten Milbrath ${ }^{2}$ \\ Vera Lucia Freitag ${ }^{3}$ \\ Ruth Irmgard Bärtschi Gabatz ${ }^{4}$ \\ Manoella Souza da Silva ${ }^{5}$ \\ Bruna Alves dos Santos ${ }^{6}$
}

${ }^{1}$ Enfermeira egressa da Universidade Federal de Pelotas (UFPel) - Rio Grande do Sul (RS). Enfermeira na Unidade de Terapia Intensiva (UTI) Pediátrica do Hospital Santo Antônio Blumenau - Santa Catarina (SC). Brasil. E-mail: karine.maciel.ecp7@gmail.com

${ }^{2}$ Enfermeira. Doutora em Enfermagem pelo Programa de Pós-graduação em Enfermagem da Universidade Federal do Rio Grande do Sul (UFRGS)

Professora Adjunta da Faculdade de Enfermagem e do Programa de Pós-graduação em Enfermagem da UFPel/RS. Brasil. E-

mail: vivianemarten@hotmail.com

${ }^{3}$ Enfermeira. Doutoranda em Enfermagem do Programa de Pós-Graduação em Enfermagem (PPGEnf) da UFRGS. Mestre em Ciências. Bolsista pela

Coordenação de Aperfeiçoamento de Pessoal de Nível Superior (CAPES). Porto Alegre/RS. Brasil. E-mail: verafreitag@hotmail.com

${ }^{4}$ Enfermeira. Doutora em Ciências pelo Programa de Pós-graduação em Enfermagem da UFPel. Professora Adjunta da Faculdade de Enfermagem da

UFPel/RS. Brasil. E-mail: r.gabatz@yahoo.com.br

${ }^{5}$ Enfermeira egressa da UFPel. Enfermeira na UTI Neonatal - Hospital Santo Antônio Blumenau/SC. Brasil. E-mail:

manoellasouza@msn.com

${ }^{6}$ Enfermeira egressa da UFPel. Enfermeira Assistente as UTI Adulto do Hospital Tacchini. Bento Gonçalves/RS. Brasil. E-mail: brunabads@hotmail.com Brazil. E-mail: annasalimena@terra.com.br.Corresponding author. 


\title{
ARTIGO ORIGINAL
}

\section{Resumo}

Trata-se de uma revisão integrativa da literatura que objetivou desvelar a produção científica dos últimos 10 anos sobre a assistência ao adulto com Síndrome da Imunodeficiência Adquirida (AIDS). Analisou-se 18 artigos, entre os anos 2008 a 2017, coletados nas bases de dados SCIELO, LILACS e BDENF. A coleta foi realizada no mês de abril de 2017, onde os artigos foram identificados por leitura dos títulos e resumos, enquanto a extração dos dados foi realizada por dois revisores. Foi feita então a análise dos dados, que foram categorizados em quadro sinóptico. Após a leitura dos artigos, foram listados para serem discutidas duas categorias temáticas: "estratégias de assistência à pessoa com AIDS" e "dificuldades enfrentadas pelos profissionais de saúde no cuidado à pessoa com AIDS". Conclui-se que o estigma social e o preconceito frente ao HIV/AIDS apresentam-se como dificuldades na prestação do cuidado integral e humanizado a pessoas. Percebe-se a necessidade de ações, como por exemplo a educação permanente no serviço de saúde, que objetivem conscientizar os profissionais da equipe multiprofissional quanto ao desvencilhamento de julgamentos e de atitudes negativas ao cuidar da pessoa que vive com esta doença crônica, garantindo assim o respeito, equidade e a prestação de uma assistência holística.

Palavras-chave: Síndrome de Imunodeficiência Adquirida; Cuidado; Doença Crônica; Equipe de Saúde.

\begin{abstract}
This is an integrative review of the literature that aimed to unveil the scientific articles of the last 10 years concerning the care for adults diagnosed with Acquired Immunodeficiency Syndrome (AIDS). We analyzed 18 articles, from 2008 to 2017, collected in the databases SCIELO, LILACS and BDENF. The research was carried out in April 2017, where the articles were identified by their titles and abstracts, while the data extraction was performed by two reviewers. The data were then analyzed and categorized in a synoptic table. After the reading of the articles, two thematic categories were discussed: "strategies to assist people with AIDS" and "difficulties faced by health professionals in the care of people living with AIDS" It is concluded that social stigma and prejudice against HIV positive people present difficulties in providing a whole and humanized care to people. It is perceived the need for actions such as permanent education in health services, which aim to make the professionals of the multiprofessional team aware of the need to get rid of judgments and negative attitudes when caring for the person living with this chronic disease, thus guaranteeing the respect, fairness and the provision of holistic assistance.
\end{abstract}

Keywords: Acquired Immunodeficiency Syndrome; Caution; Chronic disease; Health Team. 


\section{Introdução}

A Síndrome da Imunodeficiência Adquirida (AIDS) é caracterizada pela diminuição da contagem de células T CD4 +, abaixo de 350 células $\mathrm{mm} / \mathrm{mm}^{3}$, em decorrência da infecção pelo Vírus da Imunodeficiência Humana (HIV), causando o surgimento de doenças oportunistas e neoplasias características desta ${ }^{(1-2)}$.

Na perspectiva de doença crônica, a AIDS acarreta à pessoa que vive com a Síndrome da Imunodeficiência Adquirida uma mudança na rotina diária, levando-a a repensar o seu autoconceito e a sua visão sobre a sociedade em que está inserida. Além disso, a utilização de medicações diárias e a impossibilidade de cura causam sentimentos de medo e desesperança, o que interfere diretamente na adesão ao tratamento e na melhoria da qualidade de vida dessas pessoas ${ }^{(3)}$. Desta forma, a pessoa que vive com HIV/AIDS passa por um processo de descoberta relacionada à sua própria existência, gerando uma necessidade de se (re)transformar e se habituar à nova rotina ${ }^{(4)}$.

A AIDS traz, além da condição crônica, o preconceito e o estigma, o que faz com que a pessoa seja marginalizada pela sociedade em geral, que por sua vez impõem à ela a condição de culpada pela transmissão e disseminação da doença. Frente a isso, a pessoa que vive com a doença opta por manter o diagnóstico oculto até mesmo de amigos e familiares, pelo medo de sofrer repressões sociais e ter esses vínculos fragilizados ou até rompidos ${ }^{(5)}$. Dessa forma, destaca-se que os profissionais da saúde devem ter atenção à saúde mental das pessoas que vivem com AIDS, uma vez que elas podem estar em sofrimento psíquico, devido às diversas situações que vivenciam, principalmente relacionadas ao medo da rejeição afetiva, familiar e social(6).

Tendo em vista a complexidade que concerne o viver com AIDS, no que se refere à condição crônica, ao uso das medicações, ao preconceito e ao estigma social, salienta-se a importância do cuidado às pessoas que vivem com HIV/AIDS, uma vez que a enfermagem, como profissão que visa o cuidado, deve auxiliá-las no processo de enfrentamento da doença. Neste sentido, o objetivo do estudo foi desvelar a produção científica dos últimos 10 anos sobre a assistência ao adulto com síndrome da Imunodeficiência Adquirida.

\section{Método}

Trata-se de uma revisão integrativa da literatura que tem por característica o levantamento bibliográfico, realizado de maneira criteriosa, que possibilita a organização do conhecimento científico, além de aproximar o pesquisador ao tema pesquisado e sua evolução ao longo do tempo(7). Dessa forma, a revisão integrativa caracteriza-se por resumir o conhecimento, bem como sua aplicabilidade na prática $^{(8)}$.

Esta revisão integrativa foi construída respeitando seis etapas: identificação do tema e seleção da hipótese ou questão norteadora da pesquisa; estabelecimento de critérios para inclusão e exclusão de estudos; definição das informações a serem extraídas dos estudos selecionados; avaliação dos estudos incluídos na revisão; interpretação dos resultados e apresentação da revisão e síntese do conhecimento $^{(9)}$.

Para tanto, elaborou-se a seguinte questão norteadora: o que vem sendo produzido nos últimos dez anos sobre a assistência ao adulto com Síndrome da Imunodeficiência Adquirida? Foram incluídos na revisão estudos realizados com seres humanos, publicados na íntegra entre os anos de 2008 e 2017 nos idiomas inglês, português e espanhol, e que, independente do delineamento, abordaram a temática em 
destaque, estando os mesmos disponíveis nas bases de dados: Scientific Eletronic Library Online (SciELO), Literatura Latino-Americano e do Caribe de Informação em Ciências da Saúde (LILACS) e Base de Dados de Enfermagem (BDENF). Foram excluídos estudos, dissertações, monografias, revisões de literatura, estudos de caso, catálogos e cartas ao editor que não responderam a questão de pesquisa. A consulta às bases de dados foi realizada durante o mês de abril de 2017.

Os descritores utilizados para a formulação da estratégia de busca foram: "Assistência" e "Síndrome da Imunodeficiência Adquirida", sendo estes consultados previamente nos Descritores em Ciências da Saúde (DeCS). Destaca-se que foi utilizado " $A N D$ " entre os descritores, como operador booleano. No decorrer da coleta de dados, foram classificados tópicos considerados significativos, como: tipo de publicação, metodologia e amostra, local e ano de publicação, resultados apresentados com ênfase na Rede de Cuidado, dificuldades/facilidades na assistência prestada ao adulto com Síndrome Imunodeficiência Adquirida, bem como o sentimento dos profissionais frente à doença.

Após a combinação dos descritores mencionados anteriormente, e aplicados os critérios definidos, emergiram um total de 16 artigos na base de dados SciELO. Deste total foram excluídos 12 estudos pelo título não abordar a temática proposta, um por se tratar de revisão de literatura e um após a leitura do resumo, pois não foi realizado com profissionais de saúde. Assim, foram selecionados 2 artigos nessa base de dados para compor este estudo através da base de dados mencionada.

$\mathrm{Na}$ BDENF, emergiram um total de 136 artigos. Desse total, foram excluídos 87 estudos pelo título não abordar a temática proposta, 10 por se tratar de revisão de literatura, um por duplicidade com as demais bases de dados, um por ser relato de experiência e 17 após a leitura do resumo, pois não foram realizados com profissionais de saúde. Destes, restou um total de 20 estudos para leitura na íntegra. Após a análise dos textos, 7 estudos foram excluídos, pois os resultados apresentados não respondiam a questão norteadora desta pesquisa. Assim, 13 artigos foram selecionados para compor este estudo.

$\mathrm{Na}$ base LILACS, emergiram um total de 236 artigos. Desse total foram excluídos 195 estudos pelo título não abordar a temática proposta, 10 por se tratar de revisão de literatura, um por ser relato de experiência, 10 por duplicidade com as demais bases de dados e 8 após a leitura do resumo, pois não foram realizados com profissionais de saúde. Destes, restou um total de 12 estudos para leitura na íntegra. Após a análise dos textos, 9 estudos foram excluídos, pois os resultados apresentados não respondiam a questão norteadora desta pesquisa. Assim, 3 artigos foram selecionados para compor este estudo.

Dessa maneira, foram selecionados 18 artigos para compor a amostra, sendo elaborado um fluxograma para melhor compreensão da seleção.

Fluxograma 1. Fluxo de busca dos artigos selecionados para a revisão. Pelotas, RS, Brasil, 2017.

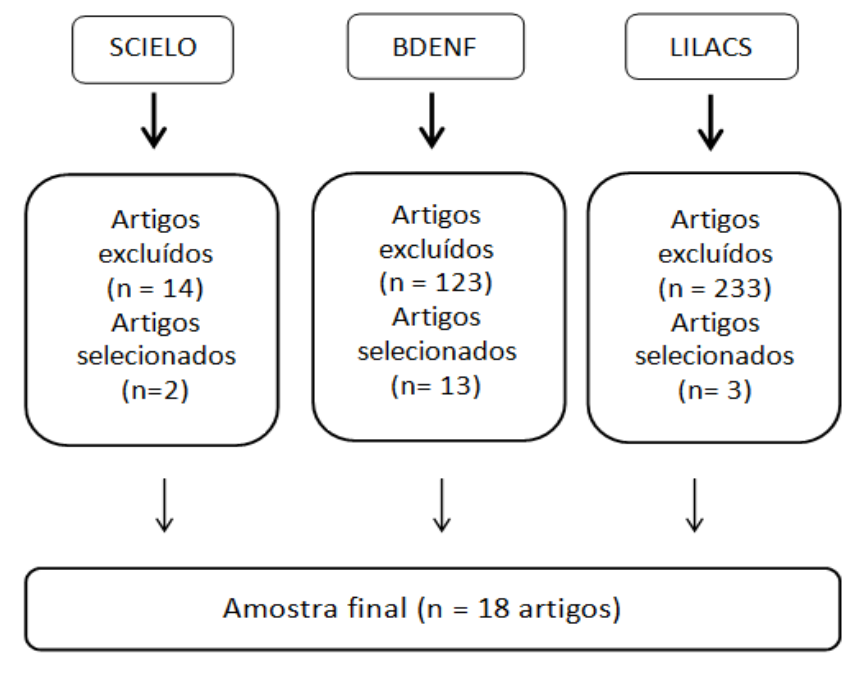

Fonte: dados da pesquisas

Resultados 
Para melhor compreensão dos estudos incluídos, as tabelas 1 e 2 demonstram a caracterização dos estudos incluídos nessa revisão. O idioma dos artigos encontrados foi, em sua maioria, português. Grande parte dos estudos foi realizada no Brasil, sendo um realizado em Portugal, e tendo como participantes, em sua maioria, somente profissionais de enfermagem, sendo os demais realizados com todos os profissionais da equipe multiprofissional.

Quanto ao local de realização das pesquisas, Hospitais públicos de referência para tratamento de HIV/AIDS, Serviço de Assistência Especializada (4), Unidades Básicas de Saúde (UBS) (2), Instituições não governamentais que prestam assistência a pessoas que vivem com HIV/AIDS (1), Secretaria Municipal de Saúde (1), Serviço de Referência para doenças infecto parasitárias (1), Ambulatório de um hospital referência pra HIV/AIDS (1), Clínica de doenças infectocontagiosas e Serviço de Assistência Especializada (1), Centro de Testagem e Serviço de Assistência Especializada (1) e Domicílio e local de trabalho dos entrevistados (1).

Quanto à abordagem metodológica, a maioria dos estudos analisados utilizou abordagem qualitativa, restando dois estudos que utilizaram abordagem quantitativa. A coleta de dados dos estudos foi realizada por meio de entrevista semiestruturada (11), análise documental e história oral temática (1), entrevista aberta e observação direta com diário de campo (1), entrevista aberta em profundidade (1), análise documental, observação de campo e entrevista semiestruturada (1), história oral (1), questionário estandartizado (1) e formulário previamente estruturado (1).

Tabela 1. Identificação dos estudos quanto ao tipo e nível de evidência. Pelotas, RS, Brasil, 2017.

\begin{tabular}{|c|c|c|}
\hline Estudo & $\begin{array}{l}\text { Tipo de } \\
\text { estudo }\end{array}$ & $\begin{array}{l}\text { Nivel de } \\
\text { evidência }\end{array}$ \\
\hline $\begin{array}{l}1 \text { Ações de promoção da saúde em serviço de assistência } \\
\text { ambulatorial especializada em HIV/AIDS (lia). }\end{array}$ & Qualitativo & NivelVI \\
\hline $\begin{array}{l}2 \text { Potencialidades e fragilidades da rede de cuidado da } \\
\text { pessoa com HIV/AIDSS(11). }\end{array}$ & Qualitativo & Nivel VI \\
\hline $\begin{array}{l}3 \text { The dimensions of nursing care provided to individuals } \\
\text { living with acquired immunodeficiency syndrome }{ }^{(2)} \text {. }\end{array}$ & Quantitativo & Nivel IV \\
\hline $\begin{array}{l}4 \text { Representaģōes sociais do cuidado prestado aos } \\
\text { pacientes soropositivos ao } \mathrm{HIV}^{144} \text {. }\end{array}$ & Qualitativo & Nivel IV. \\
\hline $\begin{array}{l}5 \text { Representaçōes de profissionais da atenção básica sobre } \\
\text { HIV/AIDS }{ }^{15} \text {. }\end{array}$ & Qualitativo & Nivel IV \\
\hline $\begin{array}{l}60 \text { cuidado a pessoas com HIV/AIDS na perspectiva de } \\
\text { profissionais de saúde }{ }^{\mid 17\}} \text {. }\end{array}$ & Qualitativo & Nivel VI \\
\hline $\begin{array}{l}7 \text { Cuidados paliativos em pacientes com HIV/AIDS: } \\
\text { principios da bioética adotados por enfermeiros }{ }^{(18)} \text {. }\end{array}$ & Qualitativo & Nivel VI \\
\hline $\begin{array}{l}8 \text { Trabalho em equipe e interdisciplinaridade: desafios } \\
\text { para a efetivaçąo da integralidade na assistência } \\
\text { ambulatorial às pessoas vivendo com HIV/AIDS em } \\
\text { Pernambuco }\end{array}$ & Qualitativo & Nivel IV \\
\hline $\begin{array}{l}9 \text { Assistência odontológica a portadores de HIV na rede de } \\
\text { serviços do sistema único de saúde em Fortaleza, Ceará }{ }^{(21)} \text {. }\end{array}$ & Quantitativo & Nivel IV \\
\hline $\begin{array}{l}10 \text { Cuidado de enfermagem em Serviço Ambulatorial } \\
\text { Especializado em HIV/AIDS }{ }^{221} \text {. }\end{array}$ & Qualitativo & Nivel VI \\
\hline $\begin{array}{l}11 \text { Percepção da AIDS pelos profissionais da saúde que } \\
\text { vivenciaram a epidemia durante o cuidado prestado às } \\
\text { pessoas com a doença, em Florianópolis (SC), Brasi|l23). }\end{array}$ & Qualitativo & Nivel VI \\
\hline $\begin{array}{l}12 \text { AIDS e as primeiras respostas voltadas para a epidemia: } \\
\text { contribuiçōes dos profissionais de saúde }{ }^{244} \text {. }\end{array}$ & Qualitativo & Nivel VI \\
\hline $\begin{array}{l}13 \text { Representaçōes sociais do HIV/AIDS por profissionais } \\
\text { de saúde em serviços de referência }\end{array}$ & Qualitativo & Nivel VI \\
\hline $\begin{array}{l}14 \text { Vulnerabilidade, empoderamento e conhecimento: } \\
\text { memórias e representaçôes de enfermeiros acerca do } \\
\text { cuidado o }^{25 .} \text {. }\end{array}$ & Qualitativo & Nivel VI \\
\hline $\begin{array}{l}15 \text { Entre sofrimento e prazer: a vulherabilidade para } \\
\text { enfermeiros nas relaçōes interpessoais com pacientes com } \\
\text { HIV/AIDS }{ }^{27]} \text {. }\end{array}$ & Qualitativo & NivelVI \\
\hline $\begin{array}{l}16 \text { Challenges and confrontations in care by nurses: a } \\
\text { study of social representation }{ }^{286} \text {. }\end{array}$ & Qualitativo & Nivel VI \\
\hline $\begin{array}{l}17 \text { Representaçōes sociais elaboradas por enfermeiros que } \\
\text { cuidam de pessoas com HV acerca de sua } \\
\text { vulherabilidade } e^{(2)} \text {. }\end{array}$ & Qualitativo & Nivel IV \\
\hline $\begin{array}{l}18 \text { Qualidade da atenção à saúde de portadores de HIV: } \\
\text { opiniäo de profissionais de saúde } \text { I }^{300} \text {. }\end{array}$ & Quantitetivo & Nivel IV \\
\hline
\end{tabular}

Fonte: dados da pesquisa.

Tabela 2. Caracterização dos estudos quanto a amostra, idioma, local e ano de publicação. Pelotas, RS, Brasil, 2017. 


\begin{tabular}{|c|c|c|c|c|}
\hline Estudo & Amostra & Idioma & Local & Ano \\
\hline 1 & $\begin{array}{l}\text { Três médicos, dois enfermeiros, dois psicólogos e } \\
\text { um odontólogo. }\end{array}$ & Português & Brasil & 2011 \\
\hline 2 & $\begin{array}{l}\text { Oito pessoas com HIV/aids e sua rede de } \\
\text { cuidado, totalizando dezoito participantes. } \\
\text { Dentre os membros da rede de cuidado familiar } \\
\text { estão: mãe, irmã, irmão e esposa. Os membros } \\
\text { da rede de cuidado institucional-profissional de } \\
\text { saúde foram representados por médicos, equipe } \\
\text { de enfermagem e psicólogos. }\end{array}$ & Português & Brasil & 2015 \\
\hline 3 & 78 profissionais de enfermagem. & Inglês & Brasil & 2016 \\
\hline 4 & 20 auxiliares de enfermagem e 20 enfermeiros. & Português & Brasil & 2010 \\
\hline 5 & Sete enfermeiras e cinco médicos. & Português & Brasil & 2009 \\
\hline 6 & 11 enfermeiros e 11 psicólogas. & Português & Portugal & 2013 \\
\hline 7 & Doze enfermeiros. & Português & Brasil & 2013 \\
\hline 8 & $\begin{array}{l}35 \text { sujeitos: os gerentes dos SAE-HIV/Aids } \\
\text { estudados (03); } 13 \text { técnicos, representantes de } \\
\text { cada categoria profissional envolvidos } \\
\text { diretamente com a assistência e que fazem parte } \\
\text { da equipe mínima proposta pelo Departamento } \\
\text { de DST, Aids e Hepatites Virass; o Coordenador } \\
\text { Estadual do Programa de DST e AIDS e o } \\
\text { Coordenador Municipal do Programa de DST e } \\
\text { Aids de Recife; e } 17 \text { usuários vinculados às } \\
\text { unidades pesquisadas. }\end{array}$ & Português & Brasil & 2012 \\
\hline 9 & 186 cirurgiōes-dentistas. & Português & Brasil & 2010 \\
\hline 10 & Dez enfermeiras. & Português & Brasil & 2016 \\
\hline 11 & $\begin{array}{l}\text { Quatro médicos, oito enfermeiras, quatro } \\
\text { técnicos de enfermagem, três auxiliares de } \\
\text { enfermagem, um dentista, um nutricionista, uma } \\
\text { assistente social e uma psicóloga. }\end{array}$ & Português & Brasil & 2014 \\
\hline 12 & Três enfermeiras e três médicos. & Português & Brasil & 2015 \\
\hline 13 & 86 profissionais de saúde. & Português & Brasil & 2014 \\
\hline 14 & 30 enfermeiros. & Português & Brasil & 2014 \\
\hline 15 & 30 enfermeiros. & Português & Brasil & 2013 \\
\hline 16 & 30 enfermeiros. & Inglês & Brasil & 2014 \\
\hline 17 & 30 enfermeiros. & Português & Brasil & 2014 \\
\hline 18 & 34 profissionais de saúde. & Português & Brasil & 2016 \\
\hline
\end{tabular}

Fonte: dados da pesquisa.

\section{Discussão}

Após a leitura dos artigos, foram listados os resultados mais abordados nas pesquisas para serem discutidos em duas categorias temáticas: "Estratégias de assistência à pessoa com Síndrome da Imunodeficiência Adquirida" e "Dificuldades enfrentadas pelos profissionais de saúde no cuidado à pessoa com Síndrome da Imunodeficiência Adquirida".

\section{Estratégias de assistência à pessoa com Síndrome da Imunodeficiência Adquirida}

Ao analisar as pesquisas realizadas, foi possível observar uma série de estratégias utilizadas para prestar assistência à pessoa com Síndrome da Imunodeficiência Adquirida. Nessas estratégias, o profissional da saúde necessita, ao cuidar de pessoas que vivem com a doença oferecer um cuidado igual ao prestado aos demais usuários, livre de preconceitos e estigmas, garantindo ao indivíduo um cuidado holístico, com base nas necessidades apresentadas por ele ${ }^{(10)}$.

Essa forma de abordagem ancora-se na perspectiva de que a pessoa que vive com AIDS vivencia um sofrimento frente ao estigma social, o que torna-se uma barreira que dificulta o acesso ao serviço, implicando diretamente na busca pela assistência devido ao medo do preconceito que sofrem diariamente ${ }^{(11)}$. Assim, torna-se essencial o desvencilhamento de julgamentos e de ações negativas por parte do profissional, com o intuito de reduzir o impacto da doença na sociedade e de aproximar a pessoa ao serviço, por meio de um acolhimento humanizado e integral ${ }^{(12)}$.

Sendo assim, um estudo destacou que é fundamental a inclusão do tema nos cursos de graduação da área da saúde, partindo do pressuposto de que o profissional em algum momento terá de cuidar de pessoas que vivem com AIDS, dando enfoque na história da doença e no impacto social. Além disso, a educação continuada é uma importante estratégia de cuidado, uma vez que proporciona a continuidade, e a troca de conhecimento e experiência entre os profissionais, sendo as informações posteriormente utilizadas com os usuários em forma de educação em 


\section{ARTIGO ORIGINAL}

saúde ${ }^{(12)}$.

Nessa conjuntura, um estudo(10) aponta que a educação em saúde apresenta-se como uma ferramenta essencial para a assistência às pessoas que vivem com AIDS, uma vez que essa tem o papel de informar a pessoa quanto aos seus direitos assistenciais e sociais. Além disso, fornece conhecimentos quanto ao cuidado de sua saúde, mudanças na rotina diária e importância da adesão ao tratamento. Assim, esse processo proporciona à pessoa empoderamento e o maior controle sobre sua vida, tornado esta a protagonista do seu cuidado, com o intuito de torna-la ativa no seu processo de cuidado(10). Em consonância, outro estudo $^{(13)}$ traz que a educação em saúde promove melhor aceitação ao tratamento antirretroviral e estreita os laços entre profissionais de saúde e a pessoa que vive com HIV/AIDS, por meio de orientações que estimulem o enfrentamento da doença.

Outra forma de assistência que se mostrou relevante foi a comunicação e a escuta ativa enquanto potencialidades no processo de cuidado à pessoa que vive com a Síndrome da Imunodeficiência Adquirida. Essas proporcionam à pessoa momentos de orientação e de conversa, assim como uma oportunidade para desabafar e aliviar sua ansiedade. Portanto, ao dar voz à pessoa com a doença, o profissional propicia um ambiente acolhedor e confortável para expressar seus sentimentos e angústias, além de consequentemente estreitar o vínculo de afetividade entre ambas as partes ao demonstrar interesse e fornecer o cuidado de forma integral, estando disponível por inteiro ${ }^{(11)}$.

O diálogo entre o profissional e a pessoa que vive com AIDS faz-se fundamental para encorajar e estimular a continuidade do tratamento, com vistas na melhoria da qualidade de vida dela(14). Em contraponto, foi analisado outro estudo(15) que discorre a respeito das dificuldades que os profissionais possuem em abordar as pessoas para intervir e estimular melhorias nos hábitos de vida das mesmas, frustrando os profissionais atuantes no serviço. Cabe salientar a importância do ato de auxiliar a população a conhecer e compreender a relevância da adoção das práticas protetivas e protetoras da infecção pelo $\mathrm{HIV}^{(16)}$, mas para tanto, os profissionais da saúde necessitam conhecer os sentidos e os significados que as pessoas que pretendem cuidar atribuem ao contrair o vírus e ao viver com ele.

Outra técnica a ser abordada é a demonstração de afetividade que é apontada por ser uma forma de sensibilização para com a situação vivida pela pessoa com a Síndrome da Imunodeficiência Adquirida(17). Assim, ao fornecer apoio e compreensão pela situação vivenciada por essas pessoas, o profissional realiza o exercício da empatia, o que o auxilia a prestar o cuidado de forma holística no momento em ela se encontra fragilizada. Além disso, o estabelecimento da relação de confiança dá ao profissional a oportunidade de conhecer e compreender o cotidiano de vida da pessoa, identificando suas necessidades físicas e psicossociais, além de prestar um cuidado mais efetivo, de acordo com a vulnerabilidade do mesmo ${ }^{(17)}$.

No que tange a comunicação, cabe ressaltar que por se tratar de uma doença estigmatizante, a pessoa que vive com HIV/AIDS tem o direito de manter o diagnóstico de forma sigilosa, cabendo ao profissional a não revelação deste para outras pessoas que participam da rede de apoio dessa, agindo assim de forma ética em sua prática profissional $^{(18)}$. Dessa forma, a ética profissional é essencial no cuidado à pessoa soropositiva, tendo em vista a preservação dos direitos desta, além da diminuição dos impactos negativos frente à estigmatização da doença na sociedade, auxiliando também na melhoria da qualidade de vida dessas pessoas $^{(19)}$.

O trabalho em equipe é descrito como uma importante estratégia no cuidado às pessoas que vivem com a Síndrome da Imunodeficiência Adquirida, pois é por meio dele que se desenvolvem ações de planejamento, avaliação e interação entre os profissionais da equipe 


\section{ARTIGO ORIGINAL}

multiprofissional no cuidado, refletindo diretamente na assistência prestada a essas ${ }^{(20)}$. Assim, é relevante oferecer assistência odontológica no cuidado integral a essas pessoas, uma vez que a manutenção bucal tem a capacidade de influenciar diretamente no comportamento da doença, evitando possíveis complicações decorrentes dela ${ }^{(21)}$.

Além disso, um estudo ${ }^{(22)}$ aborda a importância de trazer os familiares para a assistência, uma vez que eles tornam-se fundamentais ao participar ativamente no processo de cuidado, facilitando e incentivando a adesão do tratamento, e estimulando o autocuidado. Por fim, percebe-se a importância do cuidado integral e humanizado às pessoas que vivem com AIDS, sendo indispensável a constante capacitação do profissional da saúde, a fim de contribuir e garantir a qualidade da assistência prestada por meio de estratégias que aproximem o usuário do serviço e que garantam o respeito, a equidade e a prestação do cuidado humanizado, livre de atitudes negativas e preconceituosas.

\section{Dificuldades enfrentadas pelos profissionais de saúde no cuidado à pessoa com Síndrome da Imunodeficiência Adquirida}

O início da epidemia da Síndrome da Imunodeficiência Adquirida foi marcado historicamente por medo e receio, aliados a discriminação e a estigmatização da pessoa que tinha a patologia. Isso se relaciona ao fato da patologia ter acometido, inicialmente, homossexuais, usuários de drogas injetáveis e profissionais do sexo, e além de se tratar de uma doença sem cura. Em decorrência disso, muitos profissionais da saúde negaram-se a trabalhar em instituições que prestavam serviço a essas pessoas, reforçando o preconceito e a discriminação frente à doença ${ }^{(23)}$. Sabe-se que, historicamente, o estigma e o preconceito repercutiam diretamente no cuidado prestado a essas pessoas, pois os profissionais atuavam pressionados pela mídia que explorava a doença com matérias sensacionalistas que reforçavam a intolerância e o preconceito ${ }^{(24)}$.

Atualmente, o preconceito e o estigma ainda representam barreiras na prestação do cuidado, pois os profissionais trazem percepções negativas do contexto histórico para a assistência, em forma de julgamento e de culpabilização direcionados às pessoas que vivem com AIDS. Este comportamento resulta na fragilidade do vínculo estabelecido entre ambas as partes e impactando negativamente a qualidade do cuidado prestado ${ }^{(12)}$. Nessa conjuntura, ainda hoje os profissionais permanecem possuindo sentimentos negativos ao cuidar de pessoas que vivem com a Síndrome da Imunodeficiência Adquirida, uma vez que esses temem o risco de serem infectados ${ }^{(25)}$.

Segundo um estudo(26), os profissionais sentem-se vulneráveis ao cuidar de pessoas que vivem com a doença devido à falta de conhecimento e a carência de informações sobre a Síndrome da Imunodeficiência Adquirida. Assim, eles classificam o primeiro contato com o usuário como um evento traumatizante, uma vez que não possuíam conhecimentos específicos para embasar sua prática na assistência a essas pessoas. Complementarmente, os profissionais corroboram com a escassez de conhecimento para basear o cuidar de pessoas que vivem com Síndrome da Imunodeficiência Adquirida, porém relatam que a incerteza motiva a procura de meios de informações para embasar suas práticas e torná-los empoderados para o cuidar dessas pessoas ${ }^{(27)}$.

Assim, o primeiro contato do profissional com o paciente soropositivo representa um momento turbulento por se tratar de uma doença crônica que, geralmente, vem acompanhada do sofrimento experienciado pelo paciente e seus familiares ${ }^{(28)}$. Nesse sentido, o cuidado à pessoa com HIV/AIDS torna-se exaustivo devido às demandas físicas e psicológicas apresentadas por ela, uma vez que necessita de cuidados intensivos e permanentes, relacionados ao seu bem estar físico e psicossocial. O aumento da demanda, o número reduzido de profissionais especializados e a estrutura física limitada aparecem como 


\section{ARTIGO ORIGINAL}

fragilidades no cuidado à pessoa com Síndrome da Imunodeficiência Adquirida. Ao atuar em condições precárias, com um número excessivo de usuários, o profissional torna-se sobrecarregado e desestimulado, interferindo diretamente na qualidade da assistência prestada ${ }^{(11)}$.

Outro estudo ${ }^{(10)}$ aponta que as dificuldades relacionadas ao espaço físico e aos recursos humanos impactam na implementação da assistência e na promoção da saúde. Dessa forma, a falta de infraestrutura dos serviços afeta tanto os usuários, como os profissionais, uma vez que estes sentem-se desconfortáveis ao prestar o cuidado em condições precárias, tornando-os vulneráveis à situações de sobrecarga e estresse ${ }^{(29)}$. Um estudo com profissionais da saúde reforçou que estes sentem uma insatisfação relacionada à estrutura de trabalho e às condições para a realização deste, ao cuidarem da pessoa com AIDS. Porém, em sua maioria acreditam que a qualidade da assistência prestada é adequada, estando satisfeitos com o apoio prestado pelo serviço aos usuários ${ }^{(30)}$.

Nessa perspectiva, a escassez de profissionais no serviço dificulta a participação deles em ações coletivas, o que fragiliza o trabalho dentro da equipe multiprofissional. Dessa forma, ocorre a fragmentação da assistência prestada, uma vez que a equipe trabalha de forma desarticulada. Tal apoio é fortemente influenciado pelo modelo biomédico, que gira em torno das ações curativistas, o que interfere e dificulta a atenção integral aos usuários ${ }^{(20)}$. Existem ainda outras dificuldades que corroboram com a fragmentação e descentralização da assistência prestada, como a descontinuidade e a falta de planejamento das ações realizadas pelos profissionais da equipe ${ }^{(15)}$.

Além disso, os profissionais apontam dificuldades na abordagem da pessoa soropositiva pelo receio da reação desta ao receber o diagnóstico ${ }^{(27)}$. Muitas vezes, os profissionais são alvos de violência física e verbal, com agressões físicas e verbais, reações decorrentes do estado de revolta da pessoa que recebeu o diagnóstico, o que coloca os profissionais em situação de vulnerabilidade no cotidiano do cuidado da pessoa que vive com a doença. Frente a isso, observa-se a necessidade da ampliação do número de profissionais nos serviços, com vistas à melhoria da assistência prestada, uma vez que possibilitaria que eles tivessem disponibilidade de tempo para participar de reuniões de equipe, planejar o cuidado e executar o mesmo, com a diminuição da sobrecarga diária.

\section{Conclusão}

Ao responder a questão norteadora, o presente estudo identificou algumas estratégias de assistência dos profissionais de saúde no cuidado à pessoa com Síndrome da Imunodeficiência Adquirida, bem como as dificuldades encaradas por estes. O estigma social e o preconceito frente à esta doença crônica apresentam-se como dificuldades na prestação do cuidado integral e humanizado à pessoas que vivem com ela, entretanto observou-se ainda o preconceito por parte dos profissionais de saúde que muitas vezes não tem qualificação para atender a esta demanda.

Dessa maneira, percebe-se a necessidade de ações como a realização da educação permanente dentro do serviço de saúde que objetivem conscientizar os profissionais da equipe multiprofissional quanto ao desvencilhamento de julgamentos e de atitudes negativas ao cuidar da pessoa que vive com Síndrome da Imunodeficiência Adquirida, garantindo assim o respeito, equidade e a prestação de uma assistência holística.

Além disso, torna-se fundamental o trabalho conjunto entre os profissionais das diferentes áreas atuantes no serviço, a fim de consolidar o cuidado e torna-lo integral. Para isso, é importante realizar ações que permitam a troca de conhecimentos entre os membros dos serviços, visando sanar as dificuldades para cuidar de pessoas diagnosticadas com AIDS, além de possibilitar o planejamento e a continuidade da assistência prestada.

O estudo limita-se pela escassez de produção científica sobre o assunto. Todavia, este estudo traz 


\section{ARTIGO ORIGINAL}

importante contribuição para os profissionais de saúde, bem como para o ensino de graduação e pós-graduação que poderão refletir sobre a discriminação da pessoa que vive com HIV/AIDS, visto que há uma invisibilidade que silencia e gera vazio. Esta reflexão, para além do processo discriminatório, agrega conhecimento especialmente no que tange a questão da autonomia do paciente e da sua participação efetiva na assistência com foco na prevenção, implementando estratégias para diminuir o estigma em relação à vida, construindo práticas de cuidados que podem ser (re)construídas nas relações sociais e afetivas.

\section{Referências}

1. Sabino EC, Barreto CC, Sanabani SS, Focaccia R. Tratado de Infectologia. 5ed. São Paulo: Editora Atheneu, 2015. Cap. 9.1, p. 165-71.

2. Ribeiro AF, Veras MASM, Guerra MAT, Focaccia R. Tratado de Infectologia. 5áed. São Paulo: Editora Atheneu, 2015. Cap. 9.2, p. 171-86.

3. Schaurich D, Coelho DF, Motta MDGCD. A cronicidade no processo saúde-doença: repensando a epidemia da AIDS após os antiretrovirais. Revista Enfermagem UERJ. Rio de Janeiro. 2006; 14(3):455-462.

4. Almeida DP. Quando a cura não se mostra alcançável: sentidos e significados da cronicidade em um diálogo entre portadores da sida/aids e esclerose múltipla.2015. 78 p. Dissertação (Mestrado em Psicologia) Universidade Federal do Amazonas, Programa de Pós-Graduação em Psicologia, Manaus, 2015.

5. Lima FHR. A construção do preconceito no sujeito portador de HIV: o poder do discurso midiático na representação do estigma social. 2014. 132 p. Dissertação (Mestrado em Linguística Aplicada) - Centro de Humanidades, Universidade
Estadual do Ceará, Ceará, 2014.

6. Ministério da Saúde (BR). Cuidado integral às pessoas que vivem com HIV pela Atenção Básica: Manual para a equipe multiprofissional. Secretaria de Vigilância em Saúde Departamento de DST, AIDS e Hepatites Virais. Brasília: 2015. 40 p.

7. Cunha PLO. Manual Revisão Bibliográfica Sistemática Integrativa: a pesquisa baseada em evidências. Belo Horizonte: COPYRIGHT, 2014.

8. Souza MT, Silva MD, Carvalho R. Revisão integrativa: o que é e como fazer. Einstein. 2010; $8(1): 102-6$.

9. Mendes KDS, Silveira RCCC, Galvão CM. Revisão Integrativa: método pesquisa para a incorporação de evidências na saúde e na enfermagem. Texto Contexto Enfermagem. Florianópolis. 2008; 17(4):758-64.

10. Lima ICV, Galvão MTG, Paiva SS, Brito DMS. Ações de promoção da saúde em serviço de assistência ambulatorial especializada em HIV/AIDS. Cienc Cuid Saude. 2011; 10(3):556-63.

11. Tonnera LCJ, Meirelles BHS. Potencialidades e fragilidades da rede de cuidado da pessoa com HIV/AIDS. Rev Bras Enferm. 2015; 68(3):379-85.

12. Gama ASM, Ferreira DS, Oliveira DC, Gonçalves MJF. The dimensions of nursing care provided to individuals living with acquired immunodeficiency syndrome. J Nurs UFPE online. 2016; 10(10):373442.

13. Thiengo PCS, Gomes AMT, Oliveira DC. As representações do cuidado voltado à pessoa que vive com HIV/AIDS para a equipe de saúde. Rev Enfermagem Atual In Derme. 2017; 82(20):12-8.

14. Formozo GA, Oliveira DC. Representações sociais do cuidado prestado aos pacientes 


\section{ARTIGO ORIGINAL}

soropositivos ao HIV. Rev Bras Enferm. 2010; 63(2):230-7.

15. Souza MCMR, Freitas MIF. Representações de profissionais da atenção básica sobre HIV/AIDS. Rev. Min. Enferm. 2009;13(4):499-505.

16. Ayres JRCM. Práticas educativas e prevenção de HIV/AIDS: lições aprendidas e desafios atuais. Interface Comunic Saúde Educ. 2002; 6(11):11-24.

17. Sousa CSO, Silva AL. O cuidado a pessoas com HIV/AIDS na perspectiva de profissionais de saúde. Rev Esc Enferm USP. 2013; 47(4):907-14.

18. Vasconcelos MF, Costa SFG, Lopes MEL, Abrão FMS, Batista PSS, Oliveira RC. Cuidados paliativos em pacientes com HIV/AIDS: princípios da bioética adotados por enfermeiros. Ciência \& Saúde Coletiva. 2013;18(9):2559-2566.

19. Pinheiro PNC, Vieira NFC, Pereira MLD, Barroso MGT. O cuidado humano: reflexão ética acerca dos portadores do HIV/AIDS. Rev Latino-am Enfermagem. 2005;13(4):569-75.

20. Borges MIL, Sampaio AS, Gurgel IGD. Trabalho em equipe e interdisciplinaridade: desafios para a efetivação da integralidade na assistência ambulatorial às pessoas vivendo com HIV/AIDS em Pernambuco. Ciência \& Saúde Coletiva. 2012;17(1):147-56.

21. Camurça VV, Junior ANR, Camurça VV, Alencar CHM, Almeida MEL. Assistência odontológica a portadores de HIV na rede de serviços do Sistema Único de Saúde em Fortaleza, Ceará. Rev APS. 2010;13(1):18-25.

22. Macedo SM, Miranda KCL, Silveira LC, Gomes AMT. Cuidado de enfermagem em Serviço Ambulatorial Especializado em HIV/AIDS. Rev Bras Enferm. 2016; 69(3):515-21.
23. Villarinho MV, Padilha MI. Percepção da AIDS pelos profissionais da saúde que vivenciaram a epidemia durante o cuidado prestado às pessoas com a doença, em Florianópolis (SC), Brasil (19862006). Ciência \& Saúde Coletiva. 2014; 19(6):19511960.

24. Maliska ICA, Padilha MICS, Andrade SR. AIDS e as primeiras respostas voltadas para a epidemia: contribuições dos profissionais de saúde. Rev. Enferm UERJ. 2015; 23(1):15-20.

25. Dantas MS, Abrão FMS, Freitas CMSM, Oliveira DC. Representações sociais do HIV/AIDS por profissionais de saúde em serviços de referência. Rev Gaúcha Enferm. 2014; 35(4):94-100.

26. Santos El, Gomes AMT. Vulnerabilidade, empoderamento e conhecimento: memórias e representações de enfermeiros acerca do cuidado. Acta Paul Enferm. 2013;26(5):492-8.

27. Santos El, Gomes AMT, Oliveira DC, Santos CCE. Entre sofrimento e prazer: a vulnerabilidade para enfermeiros nas relações interpessoais com pacientes com HIV/AIDS. Rev. Enferm UERJ. Rio de Janeiro. 2013; 21(1):9-15.

28. Santos El, Gomes AMT, Oliveira DC, Marques SC, Bernardes MMR. Challenges and confrontations in care by nurses: a study of social representations. Online braz j nurs. 2014; 13(2):207-18.

29. Santos Él, Gomes AMT, Oliveira DC, Marques SC, Bernardes MMR, Felipe ICV. Representações sociais elaboradas por enfermeiros que cuidam de pessoas com HIV acerca de sua vulnerabilidade. Rev. Enferm UERJ. 2014; 22(3):303-8.

30. Silva RAR, Silva ITS, Costa DARS, Holanda JRR, Dantas SD, Torres GV. Qualidade da atenção à saúde de portadores de HIV: opinião de profissionais de saúde. Rev Fund Care Online. 2016; 8(4):5068-73. 